\title{
Superradiant stability of five and six-dimensional extremal Reissner-Nordstrom black holes
}

\author{
Jia-Hui Huang ${ }^{1,2,3, a} \mathbb{D}_{\mathbb{D}}$, Tian-Tian Cao ${ }^{2,3,4}$, Mu-Zi Zhang ${ }^{2,3,5}$ \\ ${ }^{1}$ Guangdong Provincial Key Laboratory of Quantum Engineering and Quantum Materials, School of Physics and Telecommunication Engineering, \\ South China Normal University, Guangzhou 510006, China \\ ${ }^{2}$ Guangdong Provincial Key Laboratory of Nuclear Science, Institute of Quantum Matter, South China Normal University, Guangzhou 510006, \\ China \\ ${ }^{3}$ Guangdong-Hong Kong Joint Laboratory of Quantum Matter, Southern Nuclear Science Computing Center, South China Normal University, \\ Guangzhou 510006, China \\ ${ }^{4}$ Zhengzhou Champion Middle School, Zhengzhou 450003, China \\ ${ }^{5}$ BinHe Experimental Middle School, Shengzhen 518000, China
}

Received: 6 July 2021 / Accepted: 3 October 2021 / Published online: 13 October 2021

(C) The Author(s) 2021

\begin{abstract}
We revisit the superradiant stability of five and six-dimensional extremal Reissner-Nordstrom black holes under charged massive scalar perturbation with a new analytical method. In each case, it is analytically proved that the effective potential experienced by the scalar perturbation has only one maximum outside the black hole horizon and no potential well exists for the superradiance modes. So the five and six-dimensional extremal Reissner-Nordstrom black holes are superradiantly stable. The new method we developed is based on the Descartes' rule of signs for the polynomial equations. Our result provides a complementary support of previous studies on the stability of higher dimensional extremal Reissner-Nordstrom black holes based on numerical methods.
\end{abstract}

\section{Introduction}

Superradiance is an interesting phenomenon in black hole physics [1-5]. When a charged bosonic wave is impinging upon a charged rotating black hole, the wave is amplified by the black hole if the wave frequency $\omega$ obeys

$\omega<n \Omega_{H}+e \Phi_{H}$,

where $e$ and $n$ are the charge and azimuthal number of the bosonic wave mode, $\Omega_{H}$ is the angular velocity of the black hole horizon and $\Phi_{H}$ is the electromagnetic potential of the black hole horizon. This superradiant scattering was studied long time ago [6-12], and has broad applications in various areas of physics(for a comprehensive review, see [4]).

a e-mail: huangjh@m.scnu.edu.cn (corresponding author)
If there is a mirror mechanism that makes the amplified wave be scattered back and forth, it will lead to the superradiant instability of the background black hole geometry [1316]. Superradiant (in)stability of various kinds of black holes has been studied extensively in the literature. The superradiant (in)stability of rotating Kerr black holes under massive scalar perturbation has been studied in [17-27]. Superradiant instability of a Kerr black hole that is perturbed by a massive vector field is also discussed in $[28,29]$. Rotating or charged black holes with asymptotically curved space are proved to be superradiantly unstable because the curved backgrounds provide natural mirror-like boundary conditions [30-41].

Among the study of superradiant (in)stability of black holes, an interesting result is that the four-dimensional extremal and non-extremal Reissner-Nordstrom (RN) black holes have been proved to be superradiantly stable against charged massive scalar perturbation in the full parameter space of the black-hole-scalar-perturbation system [42-45]. The argument is that the two conditions for superradiant instability, (i) existence of a trapping potential well outside the black hole horizon and (ii) superradiant amplification of the trapped modes, cannot be satisfied simultaneously $[42,44]$.

The stability analysis has also been extended to various higher dimensional black holes [46-55]. In Ref. [48], the asymptotically flat $\mathrm{RN}$ black holes in $D=5,6, \ldots, 11$ are shown to be stable by studying the time-domain evolution of the scalar perturbation with a numerical characteristic integration method. But higher-dimensional RN-de Sitter black holes are unstable for large values of the black hole charge and cosmological constant in $D \geqslant 7$ space-time dimensions. In Ref. [49], the authors has provided numerical evidence that 
extremal RN-de Sitter black holes are unstable for $D>6$, while asymptotically flat extremal RN black holes are stable for arbitrary $D$.

In this paper, we will develop a analytical method and prove the superradiant stability of five and six-dimensional extremal RN black holes under charged massive scalar perturbation. In Sect. 2, we give a general description of the model we are interested in. In Sects. 3 and 4, we provide the proofs for five and six-dimensional extremal RN black hole cases respectively. The last Section is devoted to conclusion and discussion.

\section{D-dimensional RN black holes and Klein-Gordon equation}

In this section we will give a description of the $D$ dimensional RN black hole and the Klein-Gordon equation for the charged massive scalar perturbation. The metric of the $D$-dimensional RN black hole $[56,57]$ is

$d s^{2}=-f(r) d t^{2}+\frac{d r^{2}}{f(r)}+r^{2} d \Omega_{D-2}^{2}$.

$d \Omega_{D-2}^{2}$ is the common line element of a $(D-2)$-dimensional unit sphere $S^{D-2}$

$d \Omega_{D-2}^{2}=d \theta_{D-2}^{2}+\sum_{i=1}^{D-3} \prod_{j=i+1}^{D-2} \sin ^{2}\left(\theta_{j}\right) d \theta_{i}^{2}$,

where the ranges of the angular coordinates are $\theta_{i} \in$ $[0, \pi](i=2, \ldots, D-2), \theta_{1} \in[0,2 \pi] . f(r)$ reads

$f(r)=1-\frac{2 m}{r^{D-3}}+\frac{q^{2}}{r^{2(D-3)}}$,

where the parameters $m$ and $q^{2}$ are related with the ADM mass $M$ and electric charge $Q$ of the RN black hole,

$m=\frac{8 \pi}{(D-2) \operatorname{Vol}\left(S^{D-2}\right)} M$,

$q=\frac{8 \pi}{\sqrt{2(D-2)(D-3)} \operatorname{Vol}\left(S^{D-2}\right)} Q$.

In the above equation, $\operatorname{Vol}\left(S^{D-2}\right)=2 \pi^{\frac{D-1}{2}} / \Gamma\left(\frac{D-1}{2}\right)$ is the volume of unit $(D-2)$-sphere. The inner and outer horizons of the RN black hole are $r_{ \pm}=\left(m \pm \sqrt{m^{2}-q^{2}}\right)^{1 /(D-3)}$. For extremal RN black holes, the inner and outer horizons become one horizon

$r_{h}=m^{1 /(D-3)}$.

The electromagnetic field outside the black hole horizon is described by the following 1 -form vector potential

$A=-\sqrt{\frac{D-2}{2(D-3)}} \frac{q}{r^{D-3}} d t=-c_{D} \frac{q}{r^{D-3}} d t$.
The equation of motion for a charged massive scalar perturbation in the RN black hole background is governed by the following covariant Klein-Gordon equation

$\left(D_{v} D^{v}-\mu^{2}\right) \phi=0$

where $D_{v}=\nabla_{v}-i e A_{v}$ is the covariant derivative and $\mu, e$ are the mass and charge of the scalar field respectively. The solution with definite angular frequency for the above KleinGordon equation can be decomposed as

$\phi\left(t, r, \theta_{i}\right)=e^{-i \omega t} R(r) \Theta\left(\theta_{i}\right)$.

The angular eigenfunctions $\Theta\left(\theta_{i}\right)$ are $(D-2)$-dimensional scalar spherical harmonics and the eigenvalues are given by $-l(l+D-3),(l=0,1,2, \ldots)[58-62]$. The radial equation is

$\Delta \frac{d}{d r}\left(\Delta \frac{d R}{d r}\right)+U R=0$,

where $\Delta=r^{D-2} f(r)$ and

$U=\left(\omega+e A_{t}\right)^{2} r^{2(D-2)}-l(l+D-3) r^{D-4} \Delta-\mu^{2} r^{D-2} \Delta$.

Define the tortoise coordinate $y$ by $d y=\frac{r^{D-2}}{\Delta} d r$ and a new radial function $\tilde{R}=r^{\frac{D-2}{2}} R$, then the radial equation (10) can be rewritten as

$\frac{d^{2} \tilde{R}}{d y^{2}}+\tilde{U} \tilde{R}=0$

where

$\tilde{U}=\frac{U}{r^{2(D-2)}}-\frac{(D-2) f(r)\left[(D-4) f(r)+2 r f^{\prime}(r)\right]}{4 r^{2}}$.

The asymptotic behaviors of $\tilde{U}$ at the spatial infinity and outer horizon are

$$
\begin{aligned}
\lim _{r \rightarrow+\infty} \tilde{U} & =\omega^{2}-\mu^{2}, \lim _{r \rightarrow r_{+}} \tilde{U} \\
& =\left(\omega-c_{D} \frac{e q}{r_{+}^{D-3}}\right)^{2}=\left(\omega-e \Phi_{h}\right)^{2},
\end{aligned}
$$

where $\Phi_{h}$ is the electric potential of the outer horizon of the black hole.

The physical boundary conditions that we need are ingoing wave at the horizon $(y \rightarrow-\infty)$ and bound states (exponentially decaying modes) at spatial infinity $(y \rightarrow+\infty)$. Then the asymptotic solutions of the Eq. (12) are as follows

$$
\begin{aligned}
& r \rightarrow+\infty(y \rightarrow+\infty), \tilde{R} \sim e^{-\sqrt{\mu^{2}-\omega^{2}} y} \\
& r \rightarrow r_{+}(y \rightarrow-\infty), \tilde{R} \sim e^{-i\left(\omega-e \Phi_{h}\right) y}
\end{aligned}
$$


The exponentially decaying modes (bound state condition) require the following inequality

$\omega^{2}<\mu^{2}$.

Next we define a new radial function $\psi=\Delta^{1 / 2} R$, then the radial equation (10) can be written as a Schrodinger-like equation

$\frac{d^{2} \psi}{d r^{2}}+\left(\omega^{2}-V\right) \psi=0$,

where $V$ is the effective potential.

In the extremal case, the explicit expression for the effective potential $V$ is

$V=\omega^{2}+\frac{B}{A}$,

where $A$ and $B$ are

$$
\begin{aligned}
A= & 4 r^{2}\left(r^{2 D-6}-2 m r^{D-3}+m^{2}\right)^{2}=4 r^{2}\left(r^{D-3}-m\right)^{4}, \\
B= & 4\left(\mu^{2}-\omega^{2}\right) r^{4 D-10}+(2 l+D-2) \\
& \times(2 l+D-4) r^{4 D-12}-8\left(m \mu^{2}-c_{D} e m \omega\right) r^{3 D-7} \\
& -4 m\left(2 \lambda_{l}+(D-4)(D-2)\right) r^{3 D-9} \\
& +4 m^{2}\left(\mu^{2}-c_{D}^{2} e^{2}\right) r^{2 D-4} \\
& +2 m^{2}\left(2 \lambda_{l}+3(D-4)(D-2)\right) r^{2 D-6} \\
& -4 m^{3}(D-4)(D-2) r^{D-3} \\
& +m^{4}(D-4)(D-2),
\end{aligned}
$$

and $\lambda_{l}=l(l+D-3)$.

The asymptotic behaviors of this effective potential $V$ at the horizon and spatial infinity are

$$
\begin{aligned}
& V \rightarrow-\infty, \quad r \rightarrow r_{h} \\
& V \rightarrow \mu^{2}, \quad r \rightarrow+\infty .
\end{aligned}
$$

At the spatial infinity, the asymptotic behavior of the derivative of the effective potential, $V^{\prime}(r)$, is

$V^{\prime}(r) \rightarrow \begin{cases}\frac{-(D-2)(D-4)-4 \lambda_{l}-8 m\left(\mu^{2}+c_{D} e \omega-2 \omega^{2}\right)}{22 r^{3}}, & D=5 ; \\ \frac{-(D-2)(D-4)-4 r_{l}}{2 r^{3}}, & D \geqslant 6 .\end{cases}$

The superradiance condition in the extremal case is

$\omega<e \Phi_{h}=e c_{D} \frac{q}{r_{h}^{D-3}}=c_{D} e=\sqrt{\frac{D-2}{2(D-3)}} e$.

Together with the bound state condition (17), we can prove $V^{\prime}(r)<0$ at spatial infinity when $D=5$. It is also obvious that $V^{\prime}(r)<0$ at spatial infinity when $D \geqslant 6$. This means that there is no potential well when $r \rightarrow+\infty$ and there is at least one extreme for the effective potential $V(r)$ outside the horizon.
In the following sections, we will prove that there is indeed only one extreme outside the event horizon $r_{h}$ for the effective potential in each case of $D=5,6$ extremal RN black holes, no potential well exists outside the event horizon for the superradiance modes. So the $D=5,6$ extremal RN black holes are superradiantly stable under massive scalar perturbation.

It is worth noting that the important mathematical theorem we will use in the proof is the Descartes' rule of signs which asserts that the number of positive roots of a polynomial equation with real coefficients is at most the number of sign changes in the sequence of the polynomial's coefficients.

\section{$3 \mathrm{D}=5$ extremal RN black holes}

For a $D=5$ extremal $\mathrm{RN}$ black hole, the event horizon is located at $r_{h}^{(5)}=\sqrt{m}$. The explicit expression of superradiance condition (25) is

$\omega<\frac{\sqrt{3}}{2} e \approx 0.87 e$.

In order to prove there is only one extreme outside the event horizon for the effective potential $V_{5}(r)$ in the $\mathrm{D}=5$ case, we will consider the derivative of $V_{5}(r)$, i.e. $V_{5}^{\prime}(r)$, and prove that there is only one real root for $V_{5}^{\prime}(r)=0$ when $r>r_{h}^{(5)}$. The key result in the proof is summarized in Table 1.

From the general expression of the effective potential (20), we can calculate that the denominator of $V_{5}^{\prime}(r)$ is $2 r^{3}\left(r^{2}-\right.$ $m)^{5}$. The numerator of $V_{5}^{\prime}(r)$ is

$$
\begin{aligned}
n_{5}= & 3 m^{5}-15 m^{4} r^{2}+2 m^{3} r^{4}(15-2 l(l+2)) \\
& +2 m^{2} r^{6}\left(-15+3 e^{2} m-4 m \mu^{2}+2 l(l+2)\right) \\
& +m r^{8}\left(15+6 e^{2} m+16 m \mu^{2}-12 \sqrt{3} e m \omega+4 l(l+2)\right) \\
& -r^{10}\left(3+8 m \mu^{2}+4 m(\sqrt{3} e-4 \omega) \omega+4 l(l+2)\right) .
\end{aligned}
$$

As mentioned before, we want to consider the real roots of $V_{5}^{\prime}(r)=0$ when $r>r_{h}^{(5)}$. It is equivalent to considering the real roots of $n_{5}(r)=0$ when $r>r_{h}^{(5)}$.

Now we make a change for the variable $r$ and let $z=$ $r^{2}-m$, then the numerator of $V_{5}^{\prime}(r)$ is rewritten as

$$
\begin{aligned}
n_{5}= & z^{5}\left(-3-4 m\left(2 \mu^{2}+(\sqrt{3} e-4 \omega) \omega\right)-4 \lambda_{l}\right) \\
& +2 m z^{4}\left(3 e^{2} m-12 m \mu^{2}-16 \sqrt{3} e m \omega+40 m \omega^{2}-8 \lambda_{l}\right) \\
& +2 m^{2} z^{3}\left(15 e^{2} m-12 m \mu^{2}-44 \sqrt{3} e m \omega+80 m \omega^{2}-10 \lambda_{l}\right) \\
& +2 m^{3} z^{2}\left(27 e^{2} m-56 \sqrt{3} e m \omega-4\left(m\left(\mu^{2}-20 \omega^{2}\right)+\lambda_{l}\right)\right) \\
& +2 m^{5} z\left(21 e^{2}-34 \sqrt{3} e \omega+40 \omega^{2}\right) \\
& +4 m^{6}\left(3 e^{2}-4 \sqrt{3} e \omega+4 \omega^{2}\right) \\
& =\sum_{i=0}^{5} a_{i} z^{i},
\end{aligned}
$$


Table 1 Possible signs of $\left\{a_{5}, a_{4}, a_{3}, a_{2}, a_{1}, a_{0}\right\}$ in different intervals of $t$

\begin{tabular}{|c|c|c|c|c|c|c|}
\hline$t$ & $a_{5}$ & $a_{4}$ & $a_{3}$ & $a_{2}$ & $a_{1}$ & $a_{0}$ \\
\hline$(0.61,0.87)$ & - & - & - & - & - & + \\
\hline$(0.41,0.61)$ & - & - & - & - & + & + \\
\hline \multirow[t]{2}{*}{$(0.25,0.41)$} & - & - & - & - & + & + \\
\hline & & & & + & & \\
\hline \multirow[t]{3}{*}{$(0.12,0.25)$} & - & - & - & - & + & + \\
\hline & & & - & + & & \\
\hline & & & + & + & & \\
\hline \multirow[t]{4}{*}{$\left(\begin{array}{ll}0, & 0.12\end{array}\right)$} & - & - & - & - & + & + \\
\hline & & - & - & + & & \\
\hline & & - & + & + & & \\
\hline & & + & + & + & & \\
\hline
\end{tabular}

where

$$
\begin{aligned}
& a_{5}=-3-8 m \mu^{2}-4 m \omega(\sqrt{3} e-4 \omega)-4 \lambda_{l}, \\
& a_{4}=2 m\left(3 e^{2} m-12 m \mu^{2}-16 \sqrt{3} e m \omega+40 m \omega^{2}-8 \lambda_{l}\right), \\
& a_{3}=2 m^{2}\left(15 e^{2} m-12 m \mu^{2}-44 \sqrt{3} e m \omega+80 m \omega^{2}-10 \lambda_{l}\right), \\
& a_{2}=2 m^{3}\left(27 e^{2} m-56 \sqrt{3} e m \omega-4\left(m\left(\mu^{2}-20 \omega^{2}\right)+\lambda_{l}\right)\right), \\
& a_{1}=2 m^{5}\left(21 e^{2}-34 \sqrt{3} e \omega+40 \omega^{2}\right), \\
& a_{0}=4 m^{6}\left(3 e^{2}-4 \sqrt{3} e \omega+4 \omega^{2}\right)=4 m^{6}(2 \omega-\sqrt{3} e)^{2} .
\end{aligned}
$$

A real root of $n_{5}(r)=0$ when $r>r_{h}^{(5)}$ corresponds to a positive root of $n_{5}(z)=0$ when $z>0$. In the next, we will prove there is indeed only one positive real root for the equation $n_{5}(z)=0$ by analyzing the signs of coefficients of $n_{5}(z)$.

It is obvious that $a_{0}>0$. Given the bound state condition and superradiance condition, $\omega^{2}<\mu^{2}, \omega<\frac{\sqrt{3}}{2} e$, it is easy to prove that $a_{5}<0$,

$$
\begin{aligned}
a_{5} & =-3-8 m \mu^{2}-4 m \omega(\sqrt{3} e-4 \omega)-4 \lambda_{l} \\
& =-3-8 m\left(\mu^{2}-\omega^{2}\right)-4 m \omega(\sqrt{3} e-2 \omega)-4 \lambda_{l}<0 .
\end{aligned}
$$

The other coefficients can be rewritten as following

$$
\begin{aligned}
a_{4}= & 2 m\left(3 e^{2} m-12 m \mu^{2}-16 \sqrt{3} e m \omega+40 m \omega^{2}-8 \lambda_{l}\right) \\
= & 2 m\left(-8 \lambda_{l}+12 m \omega^{2}-12 m \mu^{2}\right) \\
& +2 m^{2} e^{2}\left(3-16 \sqrt{3} t+28 t^{2}\right), \\
a_{3}= & 2 m^{2}\left(15 e^{2} m-12 m \mu^{2}-44 \sqrt{3} e m \omega+80 m \omega^{2}-10 \lambda_{l}\right) \\
= & 2 m^{2}\left(-10 \lambda_{l}+12 m \omega^{2}-12 m \mu^{2}\right) \\
& +2 m^{3} e^{2}\left(15-44 \sqrt{3} t+68 t^{2}\right), \\
a_{2}= & 2 m^{3}\left(27 e^{2} m-56 \sqrt{3} e m \omega-4\left(m\left(\mu^{2}-20 \omega^{2}\right)+\lambda_{l}\right)\right) \\
= & 2 m^{3}\left(-4 \lambda_{l}-4 m \mu^{2}+4 m \omega^{2}\right) \\
& +2 m^{4} e^{2}\left(27-56 \sqrt{3} t+76 t^{2}\right), \\
a_{1}= & 2 m^{5}\left(21 e^{2}-34 \sqrt{3} e \omega+40 \omega^{2}\right) \\
= & 2 m^{5} e^{2}\left(21-34 \sqrt{3} t+40 t^{2}\right),
\end{aligned}
$$

where $t=\omega / e$. For $a_{1}$, one can check $a_{1}<0$ is equivalent to

$\frac{7 \sqrt{3}}{20}<t<\frac{\sqrt{3}}{2}$, or $0.61<t<0.87$.

For $a_{2}$, the first term in (33) is negative, so a sufficient condition for $a_{2}<0$ is that the second term in (33) is also negative, i.e.

$\frac{9 \sqrt{3}}{38}<t<\frac{\sqrt{3}}{2}$, or $0.41<t<0.87$.

Using similar analysis, a sufficient condition for $a_{3}<0$ is

$\frac{5 \sqrt{3}}{34}<t<\frac{\sqrt{3}}{2}$, or $0.25<t<0.87$.

A sufficient condition for $a_{4}<0$ is

$\frac{\sqrt{3}}{14}<t<\frac{\sqrt{3}}{2}$, or $0.12<t<0.87$.

Let us analyse the signs of coefficients $\left\{a_{5}, a_{4}, a_{3}, a_{2}\right.$, $\left.a_{1}, a_{0}\right\}$ with the varying of the parameter $t$ from 0 to $\frac{\sqrt{3}}{2}$.

When $0.61<t<0.87$, according to the Eqs. (35)-(38), all $a_{i}(i=1,2,3,4)$ are negative, the signs of the six coefficients $\left\{a_{5}, a_{4}, a_{3}, a_{2}, a_{1}, a_{0}\right\}$ are $\{-----+\}$.

When $0.41<t<0.61, a_{1}$ is positive and all other coefficients are negative. The signs of the six coefficients are $\{----++\}$.

When $0.25<t<0.41$, the sign of $a_{2}$ is not fixed by the above analysis and the signs of the six coefficients may be $\{----++\}$ or $\{---+++\}$.

For further analysis, we consider following two differences,

$\frac{a_{2}}{8 m^{3}}-\frac{a_{3}}{20 m^{2}}=\frac{m e^{2}}{20}\left(105-192 \sqrt{3} t+240 t^{2}+4 \mu^{2} / e^{2}\right)$,

$\frac{a_{3}}{20 m^{2}}-\frac{a_{4}}{16 m}=\frac{3 m e^{2}}{40}\left(15-32 \sqrt{3} t+40 t^{2}+4 \mu^{2} / e^{2}\right)$. 
One can check that

$\frac{a_{2}}{8 m^{3}}>\frac{a_{3}}{20 m^{2}}$

for $0<t<0.25$, and

$\frac{a_{3}}{20 m^{2}}>\frac{a_{4}}{16 m}$

for $0<t<0.12$. This implies if $a_{3}>0$, then $a_{2}>0$ for $0<t<0.25$ and if $a_{4}>0$, then $a_{2}, a_{3}>0$ for $0<t<$ 0.12 .

When $0.12<t<0.25$, according to the Eqs. (35)(38), $a_{4}<0$ and $a_{1}>0$. The signs of $a_{2}, a_{3}$ are not fixed. But given Eq. (41), the possible signs of $\left\{a_{3}, a_{2}\right\}$ are $\{-,-\},\{-,+\},\{+,+\}$. Then the signs of the six coefficients may be $\{----++\},\{---+++\}$, or $\{--++++\}$.

When $0<t<0.12$, we can make a similar analysis as the above case and find that the signs of the six coefficients may be $\{----++\},\{---+++\},\{--++++\}$, or $\{-+++++\}$.

Based on the above analysis on the possible signs of coefficients $\left\{a_{5}, a_{4}, a_{3}, a_{2}, a_{1}, a_{0}\right\}$, we conclude that the number of sign changes in the sequence of the six coefficients is always 1 for $0<t<\frac{\sqrt{3}}{2}$. According to Descartes' rule of signs, the polynomial equation $n_{5}(z)=0$ has at most one positive real root, which means the effective potential has at most one extreme outside the horizon. And we already know that there is at least one extreme (maximum) for the effective potential outside the horizon based on the asymptotic analysis of $V_{5}(r)$. So there is only one maximum for the effective potential $V_{5}(r)$ outside the horizon and no potential well exists. The $\mathrm{D}=5$ extremal $\mathrm{RN}$ black hole is superradiantly stable.

\section{$4 \mathrm{D}=6$ extremal RN black holes}

For a $\mathrm{D}=6$ extremal $\mathrm{RN}$ black hole, the event horizon is $r_{h}^{(6)}=m^{1 / 3}$. The explicit expression of superradiance condition (25) is

$\omega<\sqrt{\frac{3}{2}} e$.

The denominator of the derivative of effective potential $V_{6}^{\prime}(r)$ is $2 r^{3}\left(r^{3}-m\right)^{5}$. The numerator of $V_{6}^{\prime}(r)$ is

$$
\begin{aligned}
n_{6}= & -4\left(\lambda_{l}+2\right) r^{15}+4\left(-3 m \mu^{2}-\sqrt{6} e m \omega+6 m \omega^{2}\right) r^{14} \\
& +40 m r^{12} \\
& +4\left(2 e^{2} m^{2}+6 m^{2} \mu^{2}-3 \sqrt{6} e m^{2} \omega\right) r^{11} \\
& +4 m^{2}\left(-20+3 \lambda_{l}\right) r^{9} \\
& +4 m^{3}\left(2 e^{2}-3 \mu^{2}\right) r^{8}+8 m^{3}\left(10-\lambda_{l}\right) r^{6} \\
& -40 m^{4} r^{3}+8 m^{5}
\end{aligned}
$$

One can check that when $r$ goes to infinity, the asymptotic behavior of $V_{6}^{\prime}(r)$ is

$V_{6}^{\prime} \rightarrow \frac{-2\left(\lambda_{l}+2\right)}{r^{3}}+\mathcal{O}\left(\frac{1}{r^{4}}\right)$

which is negative. Thus, there is no trapping potential well near the spatial infinity. This result is consistent with the general discussion before.

Now we change the radial variable from $r$ to $z=r-r_{h}^{(6)}=$ $r-m^{1 / 3}$. The numerator of the derivative of the effective potential, $n_{6}$, is rewritten as

$$
\begin{aligned}
n^{(6)}(z)= & \left.-8-4 \lambda_{l}\right) z^{15}+4\left(-30 m^{1 / 3}-3 m \mu^{2}\right. \\
& \left.-\sqrt{6} e m \omega+6 m \omega^{2}-15 m^{1 / 3} \lambda_{l}\right) z^{14} \\
& +4\left(-210 m^{2 / 3}-42 m^{4 / 3} \mu^{2}-14 \sqrt{6} e m^{4 / 3} \omega\right. \\
& \left.+84 m^{4 / 3} \omega^{2}-105 m^{2 / 3} \lambda_{l}\right) z^{13} \\
& +4\left(-900 m-273 m^{5 / 3} \mu^{2}-91 \sqrt{6} e m^{5 / 3} \omega\right. \\
& \left.+546 m^{5 / 3} \omega^{2}-455 m \lambda_{l}\right) z^{12} \\
& +4\left(-2610 m^{4 / 3}+2 e^{2} m^{2}-1086 m^{2} \mu^{2}\right. \\
& -367 \sqrt{6} e m^{2} \omega \\
& \left.+2184 m^{2} \omega^{2}-1365 m^{4 / 3} \lambda_{l}\right) z^{11} \\
& +4\left(-5346 m^{5 / 3}+22 e^{2} m^{7 / 3}-2937 m^{7 / 3} \mu^{2}\right. \\
& -1034 \sqrt{6} e m^{7 / 3} \omega \\
& \left.+6006 m^{7 / 3} \omega^{2}-3003 m^{5 / 3} \lambda_{l}\right) z^{10} \\
& +4\left(-7830 m^{2}+110 e^{2} m^{8 / 3}-5676 m^{8 / 3} \mu^{2}\right. \\
& +2167 \sqrt{6} e m^{8 / 3} \omega \\
& \left.+12012 m^{8 / 3} \omega^{2}-5002 m^{2} \lambda_{l}\right) z^{9} \\
& +4\left(-8100 m^{7 / 3}+332 e^{2} m^{3}-8022 m^{3} \mu^{2}\right. \\
& -3498 \sqrt{6} e m^{3} \omega \\
& \left.+18018 m^{3} \omega^{2}-6408 m^{7 / 3} \lambda_{l}\right) z^{8} \\
& +4\left(-5670 m^{8 / 3}+676 e^{2} m^{10 / 3}\right. \\
& \left.-20592 m^{10 / 3} \omega^{2}-6327 m^{8 / 3} \lambda_{l}\right) z^{7} \\
& +4 / 3 \mu^{2}-4422 \sqrt{6} e m^{10 / 3} \omega \\
& \\
&
\end{aligned}
$$




$$
\begin{aligned}
& +4\left(-2430 m^{3}+980 e^{2} m^{11 / 3}-6321 m^{11 / 3} \mu^{2}\right. \\
& -4389 \sqrt{6} e m^{11 / 3} \omega \\
& \left.+18018 m^{11 / 3} \omega^{2}-4755 m^{3} \lambda_{l}\right) z^{6} \\
& +4\left(-486 m^{10 / 3}+1036 e^{2} m^{4}\right. \\
& -3402 m^{4} \mu^{2}-3388 \sqrt{6} e m^{4} \omega \\
& \left.+12012 m^{4} \omega^{2}-2637 m^{10 / 3} \lambda_{l}\right) z^{5} \\
& +4\left(800 e^{2} m^{13 / 3}-1233 m^{13 / 3} \mu^{2}\right. \\
& +1991 \sqrt{6} e m^{13 / 3} \omega \\
& \left.+6006 m^{13 / 3} \omega^{2}-1017 m^{11 / 3} \lambda_{l}\right) z^{4} \\
& +4\left(442 e^{2} m^{14 / 3}-270 m^{14 / 3} \mu^{2}-859 \sqrt{6} e m^{14 / 3} \omega\right. \\
& +8 m^{17 / 3}(\sqrt{2} e-\sqrt{3} \omega)^{2} \\
& +4\left(38 e^{2} m^{16 / 3}-47 \sqrt{6} e m^{16 / 3} \omega\right. \\
& \left.+2184 m^{14 / 3} \omega^{2}-243 m^{4} \lambda_{l}\right) z^{3} \\
& +4\left(166 e^{2} m^{5}-27 m^{5} \mu^{2}-256 \sqrt{6} e m^{5} \omega\right. \\
& \left.+546 m^{5} \omega^{2}-27 m^{13 / 3} \lambda_{l}\right) z^{2} \\
& +0 \\
& +
\end{aligned}
$$

We will prove that the polynomial equation $n_{6}(z)=0$ has at most one positive real root in the following. According to Descartes' rule of signs, we need to prove the number of sign changes is 1 in the sequence of the polynomial's sixteen coefficients, $\left\{b_{15}, b_{14}, \ldots, b_{0}\right\}$.

It is easy to see that

$b_{0}=8 m^{17 / 3}(\sqrt{2} e-\sqrt{3} \omega)^{2}>0, \quad b_{15}=-8-4 \lambda_{l}<0$.

Let's check the sign of $b_{14}$. We rewrite $b_{14}$ as following

$$
\begin{aligned}
b_{14}= & 4\left(-30 m^{1 / 3}-3 m \mu^{2}-\sqrt{6} e m \omega+6 m \omega^{2}-15 m^{1 / 3} \lambda_{l}\right) \\
= & 4\left[-30 m^{1 / 3}-15 m^{1 / 3} \lambda_{l}+\left(-3 m \mu^{2}+3 m \omega^{2}\right)\right. \\
& \left.+\left(3 m \omega^{2}-\sqrt{6} e m \omega\right)\right] .
\end{aligned}
$$

Taking into account the bound state and superradiance conditions, $\omega<\mu, \omega<e \Phi_{h}=\sqrt{2 / 3} e$, the two terms in parentheses of the above equation are negative and then

$b_{14}<0$.
Similarly, we can easily prove

$b_{13}<0, \quad b_{12}<0$.

However, it is not easy to prove the signs of $b_{1}, b_{2}, \ldots, b_{11}$ similarly.

Let's study the relation between the signs of $b_{1}$ and $b_{2}$. These two coefficients can be read out directly from (46),

$$
\begin{aligned}
b_{1}= & 4\left(38 e^{2} m^{16 / 3}-47 \sqrt{6} e m^{16 / 3} \omega+84 m^{16 / 3} \omega^{2}\right), \\
b_{2}= & 4\left(166 e^{2} m^{5}-27 m^{5} \mu^{2}-256 \sqrt{6} e m^{5} \omega\right. \\
& \left.+546 m^{5} \omega^{2}-27 m^{13 / 3} \lambda_{l}\right) .
\end{aligned}
$$

Define two new coefficients $b_{1}^{\prime}, b_{2}^{\prime}$,

$b_{1}^{\prime}=\frac{b_{1}}{4 * 84 m^{16 / 3}}, b_{2}^{\prime}=\frac{b_{2}}{4 * 519 m^{5}}$.

The difference between $b_{1}^{\prime}$ and $b_{2}^{\prime}$ is

$$
\begin{aligned}
b_{1}^{\prime}-b_{2}^{\prime}= & \frac{321 e^{2}}{2422}+\frac{256}{173} \sqrt{\frac{2}{3}} e \omega-\frac{47 e \omega}{14 \sqrt{6}} \\
& +\frac{9 \lambda_{l}}{173 m^{2 / 3}}+\frac{9}{173}\left(\mu^{2}-\omega^{2}\right) .
\end{aligned}
$$

Given the bound state condition, $\omega^{2}<\mu^{2}$, the last term in the above equation is positive. Given the superradiance condition (43), one can check the sum of the first three terms in the above equation is positive. It is obvious that the $\lambda_{l}$ term in the above equation is also positive. So we have

$b_{1}^{\prime}-b_{2}^{\prime}>0$.

The possible signs of $\left\{b_{2}, b_{1}\right\}$ is $\{-,-\},\{-,+\},\{+,+\}$. The sign of $b_{1}$ is not smaller than the sign of $b_{2}$, i.e.

$\operatorname{sign}\left(b_{2}\right) \leqslant \operatorname{sign}\left(b_{1}\right)$.

Next, let's study the relation between the signs of $b_{2}$ and $b_{3}$. The coefficient $b_{3}$ can be read out directly from (46),

$$
\begin{aligned}
b_{3}= & 4\left(442 e^{2} m^{14 / 3}-270 m^{14 / 3} \mu^{2}\right. \\
& \left.-859 \sqrt{6} e m^{14 / 3} \omega+2184 m^{14 / 3} \omega^{2}-243 m^{4} \lambda_{l}\right) .
\end{aligned}
$$

Define a new coefficient $b_{3}^{\prime}$,

$b_{3}^{\prime}=\frac{b_{3}}{7656 m^{14 / 3}}$.

The difference between $b_{2}^{\prime}$ and $b_{3}^{\prime}$ is

$$
\begin{aligned}
b_{2}^{\prime}-b_{3}^{\prime}= & \frac{4907 e^{2}}{55187}-\frac{256}{173} \sqrt{\frac{2}{3}} e \omega+\frac{859 e \omega}{319 \sqrt{6}} \\
& +\frac{8271 \lambda_{l}}{110374 m^{2 / 3}}+\frac{4914}{55187}\left(\mu^{2}-\omega^{2}\right) .
\end{aligned}
$$

Given the bound state condition, $\omega^{2}<\mu^{2}$, the last term in the above equation is positive. Given the superradiance condition (43), one can check the sum of the first three terms 
in the above equation is positive. It is obvious that the $\lambda_{l}$ term in the above equation is also positive. So we have

$b_{2}^{\prime}-b_{3}^{\prime}>0$.

The possible signs of $\left\{b_{3}, b_{2}\right\}$ is $\{-,-\},\{-,+\},\{+,+\}$. The sign of $b_{2}$ is not smaller than the sign of $b_{3}$, i.e.

$\operatorname{sign}\left(b_{3}\right) \leqslant \operatorname{sign}\left(b_{2}\right)$.

In order to study the relation between the signs of $b_{3}$ and $b_{4}$. Define a new parameter

$b_{4}^{\prime}=\frac{b_{4}}{4 * 4473 m^{13 / 3}}$.

The difference between $b_{3}^{\prime}$ and $b_{4}^{\prime}$ is

$$
\begin{aligned}
b_{3}^{\prime}-b_{4}^{\prime}= & \frac{32137 e^{2}}{507529}+\frac{1991 \sqrt{\frac{2}{3}} e \omega}{1591}-\frac{859 e \omega}{319 \sqrt{6}} \\
& +\frac{87411 \lambda_{l}}{1015058 m^{2 / 3}}+\frac{59514}{507529}\left(\mu^{2}-\omega^{2}\right) .
\end{aligned}
$$

We can prove the above difference is positive with the same method as previous cases. So the possible signs of $\left\{b_{4}, b_{3}\right\}$ is $\{-,-\},\{-,+\},\{+,+\}$, i.e.

$\operatorname{sign}\left(b_{4}\right) \leqslant \operatorname{sign}\left(b_{3}\right)$.

Similarly, define the following new coefficients

$$
\begin{aligned}
b_{5}^{\prime} & =\frac{b_{5}}{4 * 8610 m^{4}}, b_{6}^{\prime}=\frac{b_{6}}{4 * 11697 m^{11 / 3}}, \\
b_{7}^{\prime} & =\frac{b_{7}}{4 * 12252 m^{10 / 3}}, \\
b_{8}^{\prime} & =\frac{b_{8}}{39984 m^{3}}, b_{9}^{\prime}=\frac{b_{9}}{25344 m^{8 / 3}}, \\
b_{10}^{\prime} & =\frac{b_{10}}{12276 m^{7 / 3}}, b_{11}^{\prime}=\frac{b_{11}}{4392 m^{2}} .
\end{aligned}
$$

Given the bound state condition, $\omega^{2}<\mu^{2}$, and the superradiance condition (43), we find that [see the Appendix]

$b_{4}^{\prime}>b_{5}^{\prime}>b_{6}^{\prime}>b_{7}^{\prime}>b_{8}^{\prime}>b_{9}^{\prime}>b_{10}^{\prime}>b_{11}^{\prime}$.

So we have

$$
\begin{aligned}
& \operatorname{sign}\left(b_{11}\right) \leqslant \operatorname{sign}\left(b_{10}\right) \leqslant \operatorname{sign}\left(b_{9}\right) \leqslant \operatorname{sign}\left(b_{8}\right) \\
& \leqslant \operatorname{sign}\left(b_{7}\right) \leqslant \operatorname{sign}\left(b_{6}\right) \leqslant \operatorname{sign}\left(b_{5}\right) \leqslant \operatorname{sign}\left(b_{4}\right) .
\end{aligned}
$$

With the results (57), (62), (65),(69), the possible signs of $\left\{b_{11}, \ldots, b_{1}\right\}$ may be all plus, $\{+,+, \ldots,+,+\}$, all minus $\{-,-, \ldots,-,-\}$, or $\{-, \ldots,-,+, \ldots,+\}$. The signs of the sixteen coefficients of $n_{6}(z),\left\{b_{15}, b_{14}, b_{13}, \ldots, b_{1}, b_{0}\right\}$, are $\{-,-,-,-, *, *, \ldots, *,+\}$. The $*$ parts are the signs of $\left\{b_{11}, \ldots, b_{1}\right\}$. It is obvious that for all possible signs of $\left\{b_{11}, \ldots, b_{1}\right\}$, the number of the sign changes in the sequence of the sixteen coefficients is always 1 .

So there is at most one extreme for the effective potential $V_{6}(r)$ outside the horizon and together with our previous asymptotic analysis of $V_{6}(r)$, we conclude that there is only one maximum for the effective potential $V_{6}(r)$ outside the horizon and no potential well exists. The $\mathrm{D}=6$ extremal RN black hole is superradiantly stable.

\section{Conclusion and discussion}

In this paper, superradiant stability of $\mathrm{D}=5,6$ extremal $\mathrm{RN}$ black holes under charged massive scalar perturbation is revisited. A new analytical method is developed that depends mainly on Descartes' rule of signs for the polynomial equations. In $D=5$ case, based on the asymptotic analysis of the effective potential $V(r)$ in (24), we know there is at least one extreme for the effective potential outside the horizon. With the new method, we prove that the derivative of the effective potential has at most one extreme outside the horizon in Table 1. There is only one maximum for the effective potential outside the horizon and no potential well exists, so the extremal RN black hole is superradiantly stable. In $D=6$ case, the asymptotic analysis (45) shows that the effective potential has at least one extreme outside the horizon. According to the sign relations (57), (62), (65),(69) and Descartes' rule of signs, we know there is at most one extreme for the effective potential outside the horizon. There is only one maximum for the effective potential outside the horizon and no potential well exists, so the six-dimensional extremal RN black hole is also superradiantly stable.

Our result provides a complementary analytical proof for the previous work $[48,49]$ in $D=5,6$ cases. In $D=6$ case, it is quite unexpected that there are such interesting sign relations (57), (62), (65),(69) between the signs of the complicated coefficients in $n_{6}(z)$. It does not seem to be a coincidence. Due to this observation, we also have finished part of the proof for $D=7$ case and found similar interesting sign relations. So, with the new method, it is likely to provide an analytical proof that all $D$-dimensional $(D \geqslant 5)$ extremal RN black holes are superradiantly stable under charged massive scalar perturbation which is minimally coupled with the black holes, although it is not an easy work.

Acknowledgements This work is partially supported by Guangdong Major Project of Basic and Applied Basic Research (no. 2020B0301030008), Science and Technology Program of Guangzhou (no. 2019050001) and Natural Science Foundation of Guangdong Province (no. 2020A1515010388, no. 2020A1515010794).

Data Availability Statement This manuscript has no associated data or the data will not be deposited. [Authors' comment: Our study is almost analytically performed and all the related formulas have been presented in the manuscript. There is no associated data in this manuscript.]

Open Access This article is licensed under a Creative Commons Attribution 4.0 International License, which permits use, sharing, adaptation, distribution and reproduction in any medium or format, as long as you give appropriate credit to the original author(s) and the source, pro- 
vide a link to the Creative Commons licence, and indicate if changes were made. The images or other third party material in this article are included in the article's Creative Commons licence, unless indicated otherwise in a credit line to the material. If material is not included in the article's Creative Commons licence and your intended use is not permitted by statutory regulation or exceeds the permitted use, you will need to obtain permission directly from the copyright holder. To view a copy of this licence, visit http://creativecomm ons.org/licenses/by/4.0/.

Funded by SCOAP ${ }^{3}$.

\section{Appendix}

In this appendix, we will present the mentioned details in the proof of $D=6$ case. Define the following scaled coefficients

$$
\begin{aligned}
b_{5}^{\prime} & =\frac{b_{5}}{4 * 8610 m^{4}}, b_{6}^{\prime}=\frac{b_{6}}{4 * 11697 m^{11 / 3}}, \\
b_{7}^{\prime} & =\frac{b_{7}}{4 * 12252 m^{10 / 3}}, \\
b_{8}^{\prime} & =\frac{b_{8}}{39984 m^{3}}, b_{9}^{\prime}=\frac{b_{9}}{25344 m^{8 / 3}}, \\
b_{10}^{\prime} & =\frac{b_{10}}{12276 m^{7 / 3}}, b_{11}^{\prime}=\frac{b_{11}}{4392 m^{2}} .
\end{aligned}
$$

We have the following differences

$$
\begin{aligned}
& b_{4}^{\prime}-b_{5}^{\prime}=\frac{15422 e^{2}}{326155}-\frac{7711 \sqrt{6} e \omega}{326155}+\frac{425559 \lambda_{l}}{4566170 m^{2 / 3}} \\
& +\frac{81}{1435 m^{2 / 3}}+\frac{44616}{326155}\left(\mu^{2}-\omega^{2}\right), \\
& b_{5}^{\prime}-b_{6}^{\prime}=\frac{12518 e^{2}}{342555}-\frac{242}{205} \sqrt{\frac{2}{3}} e \omega \\
& +\frac{209}{557} \sqrt{6} e \omega+\frac{160247 \lambda_{l}}{1598590 m^{2 / 3}} \\
& +\frac{120933}{799295 m^{2 / 3}}+\frac{16588}{114185}\left(\mu^{2}-\omega^{2}\right), \\
& b_{6}^{\prime}-b_{7}^{\prime}=\frac{16269 e^{2}}{568697}-\frac{209}{557} \sqrt{6} e \omega \\
& +\frac{737 \sqrt{\frac{3}{2}} e \omega}{1021}+\frac{1749851 \lambda_{l}}{15923516 m^{2 / 3}} \\
& +\frac{2030535}{7961758 m^{2 / 3}}+\frac{79794}{568697}\left(\mu^{2}-\omega^{2}\right), \\
& b_{7}^{\prime}-b_{8}^{\prime}=\frac{18678 e^{2}}{850493}-\frac{9339 \sqrt{6} e \omega}{850493}+\frac{424059 \lambda_{l}}{3401972 m^{2 / 3}} \\
& +\frac{591165}{1700986 m^{2 / 3}}+\frac{29601}{242998}\left(\mu^{2}-\omega^{2}\right), \\
& b_{8}^{\prime}-b_{9}^{\prime}=\frac{3803 e^{2}}{239904}-\frac{583}{833} \sqrt{\frac{3}{2}} e \omega+\frac{197 e \omega}{96 \sqrt{6}}+\frac{391621 \lambda_{l}}{2638944 m^{2 / 3}} \\
& +\frac{124755}{293216 m^{2 / 3}}+\frac{533}{5712}\left(\mu^{2}-\omega^{2}\right) \\
& b_{9}^{\prime}-b_{10}^{\prime}=\frac{91 e^{2}}{8928}+\frac{94}{93} \sqrt{\frac{2}{3}} e \omega-\frac{197 e \omega}{96 \sqrt{6}}+\frac{18565 \lambda_{l}}{98208 m^{2 / 3}} \\
& +\frac{5523}{10912 m^{2 / 3}}+\frac{91}{1488}\left(\mu^{2}-\omega^{2}\right) \\
& b_{10}^{\prime}-b_{11}^{\prime}=\frac{91 e^{2}}{17019}-\frac{94}{93} \sqrt{\frac{2}{3}} e \omega+\frac{367 e \omega}{183 \sqrt{6}}+\frac{1001 \lambda_{l}}{3782 m^{2 / 3}}
\end{aligned}
$$

$$
+\frac{1201}{1891 m^{2 / 3}}+\frac{182}{5673}\left(\mu^{2}-\omega^{2}\right) .
$$

One can easily check that all the differences are positive under bound state and superradiance conditions.

\section{References}

1. C.A. Manogue, Ann. Phys. 181, 261 (1988)

2. W. Greiner, B. Muller, J. Rafelski, Quantum Electrodynamics of Strong Fields (Springer, Berlin, 1985)

3. V. Cardoso, O.J.C. Dias, J.P.S. Lemos, S. Yoshida, Phys. Rev. D 70, 044039 (2004).

4. R. Brito, V. Cardoso, P. Pani, Lect. Notes Phys. 906, 1 (2015)

5. R. Brito, V. Cardoso, P. Pani, Class. Quantum Gravity 32(13), 134001 (2015)

6. R. Penrose, Rev. Del Nuovo Cimento 1, 252 (1969)

7. D. Christodoulou, Phys. Rev. Lett. 25, 1596 (1970)

8. C.W. Misner, Phys. Rev. Lett. 28, 994 (1972)

9. Y.B. Zeldovich, JETP Lett. 14, 180 (1971)

10. J.M. Bardeen, W.H. Press, S.A. Teukolsky, Astrophys. J. 178, 347 (1972)

11. J.D. Bekenstein, Phys. Rev. D 7, 949 (1973)

12. T. Damour, N. Deruelle, R. Ruffini, Lett. Nuovo Cim. 15, 257 (1976)

13. W.H. Press, S.A. Teukolsky, Nature (London) 238, 211 (1972)

14. V. Cardoso, O.J.C. Dias, J.P.S. Lemos, S. Yoshida, Phys. Rev. D 70, 044039 (2004).

15. C.A.R. Herdeiro, J.C. Degollado, H.F. Runarsson, Phys. Rev. D 88, 063003 (2013)

16. J.C. Degollado, C.A.R. Herdeiro, Phys. Rev. D 89(6), 063005 (2014)

17. M.J. Strafuss, G. Khanna, Phys. Rev. D 71, 024034 (2005)

18. R.A. Konoplya, A. Zhidenko, Phys. Rev. D 73, 124040 (2006)

19. V. Cardoso, S. Chakrabarti, P. Pani, E. Berti, L. Gualtieri, Phys. Rev. Lett. 107, 241101 (2011)

20. S.R. Dolan, Phys. Rev. D 87(12), 124026 (2013)

21. S. Hod, Phys. Lett. B 708, 320 (2012)

22. S. Hod, Phys. Lett. B 736, 398 (2014)

23. A.N. Aliev, JCAP 1411(11), 029 (2014)

24. S. Hod, Phys. Lett. B 758, 181 (2016)

25. J.C. Degollado, C.A.R. Herdeiro, E. Radu, Phys. Lett. B 781, 651 (2018)

26. J.H. Huang, W.X. Chen, Z.Y. Huang, Z.F. Mai, Phys. Lett. B 798, 135026 (2019)

27. S. Ponglertsakul, B. Gwak, Eur. Phys. J. C 80(11), 1023 (2020)

28. W.E. East, F. Pretorius, Phys. Rev. Lett. 119(4), 041101 (2017)

29. W.E. East, Phys. Rev. D 96(2), 024004 (2017)

30. V. Cardoso, O.J.C. Dias, Phys. Rev. D 70, 084011 (2004)

31. V. Cardoso, O.J.C. Dias, G.S. Hartnett, L. Lehner, J.E. Santos, JHEP 1404, 183 (2014)

32. C.Y. Zhang, S.J. Zhang, B. Wang, JHEP 1408, 011 (2014)

33. O. Delice, T. Durgut, Phys. Rev. D $92(2), 024053$ (2015)

34. A.N. Aliev, Eur. Phys. J. C 76(2), 58 (2016)

35. M. Wang, C. Herdeiro, Phys. Rev. D 93(6), 064066 (2016)

36. H.R.C. Ferreira, C.A.R. Herdeiro, Phys. Rev. D 97(8), 084003 (2018)

37. M. Wang, C. Herdeiro, Phys. Rev. D 89(8), 084062 (2014)

38. P. Bosch, S.R. Green, L. Lehner, Phys. Rev. Lett. 116(14), 141102 (2016)

39. Y. Huang, D.J. Liu, X.Z. Li, Int. J. Mod. Phys. D 26(13), 1750141 (2017)

40. P.A. Gonzalez, E. Papantonopoulos, J. Saavedra, Y. Vasquez, Phys. Rev. D 95(6), 064046 (2017) 
41. Z. Zhu, S.J. Zhang, C.E. Pellicer, B. Wang, E. Abdalla, Phys. Rev. D 90(4), 044042 (2014).

42. S. Hod, Phys. Lett. B 713, 505-508 (2012)

43. J.H. Huang, Z.F. Mai, Eur. Phys. J. C 76(6), 314 (2016)

44. S. Hod, Phys. Rev. D 91(4), 044047 (2015)

45. L. Di Menza, J.-P. Nicolas, Class. Quantum Gravity 32(14), 145013 (2015)

46. R.A. Konoplya, A. Zhidenko, Rev. Mod. Phys. 83, 793-836 (2011)

47. R.A. Konoplya, A. Zhidenko, Nucl. Phys. B 777, 182-202 (2007)

48. R.A. Konoplya, A. Zhidenko, Phys. Rev. Lett. 103, 161101 (2009)

49. R.A. Konoplya, A. Zhidenko, Phys. Rev. D 89(2), 024011 (2014)

50. R.A. Konoplya, A. Zhidenko, Phys. Rev. D 78, 104017 (2008)

51. A. Ishibashi, H. Kodama, Prog. Theor. Phys. 110, 901-919 (2003)

52. H. Kodama, A. Ishibashi, Prog. Theor. Phys. 111, 29-73 (2004)
53. H. Kodama, Prog. Theor. Phys. Suppl. 172, 11-20 (2008)

54. A. Ishibashi, H. Kodama, Prog. Theor. Phys. Suppl. 189, 165-209 (2011)

55. H. Ishihara, M. Kimura, R.A. Konoplya, K. Murata, J. Soda, A. Zhidenko, Phys. Rev. D 77, 084019 (2008)

56. R.C. Myers, M.J. Perry, Ann. Phys. 172, 304 (1986)

57. K. Destounis, Phys. Rev. D 100(4), 044054 (2019)

58. A. Chodos, E. Myers, Ann. Phys. 156, 412 (1984)

59. A. Higuchi, J. Math. Phys. 28, 1553 (1987).

60. M.A. Rubin, C.R. Ordonez, J. Math. Phys. 25, 2888 (1984)

61. J.B. Achour, E. Huguet, J. Queva, J. Renaud, J. Math. Phys. 57(2), 023504 (2016)

62. L. Lindblom, N.W. Taylor, F. Zhang, Gen. Relativ. Gravit. 49(11), 139 (2017) 